\title{
A Search for Bright Kuiper Belt Objects
}

\author{
Michael J. I. Brown and R. L. Webster \\ School of Physics, University of Melbourne, Parkville, Vic. 3052, Australia \\ mbrown@physics.unimelb.edu.au \\ Received 1997 September 2, accepted 1998 April 20
}

\begin{abstract}
Since 1992, 60 large Kuiper Belt objects have been detected by ground-based telescopes. Previous surveys which have detected objects have searched approximately $60 \square^{\circ}$ and detected objects with magnitudes $20 \cdot 6<m_{R}<25 \cdot 0$. However, the luminosity function of brighter Kuiper Belt objects is not well determined. The detection of brighter objects would improve our ability to determine the Kuiper Belt objects' surface composition and provide constraints on the population statistics of different formation mechanisms. This paper describes a survey of $12 \cdot 0 \square^{\circ}$ of sky near the ecliptic to a limiting magnitude of $m_{R} \sim 21$. A slow moving candidate was detected near the magnitude limit of the survey.
\end{abstract}

Keywords: comets: general—-solar system: general

\section{Introduction}

Since the discovery of $1_{1992 Q_{1}}$ (Jewitt \& Luu 1993), a total of 60 large ( $>50 \mathrm{~km}$ radius) but dim $\left(m_{R}>20 \cdot 6\right)$ objects have been found with orbits beyond Neptune (Marsden 1997). It is thought that these objects are members of a belt of objects beyond Neptune (the Kuiper Belt) hypothesised by Edgeworth (1949) and Kuiper (1951).

Previous published surveys for Kuiper Belt objects are summarised in Table 1. Successful surveys for Kuiper Belt objects have covered a total area of $\sim 60 \square^{\circ}$ near the ecliptic (Jewitt \& Luu 1995; Williams et al. 1995; Irwin, Tremaine \& Żytkow 1995; Jewitt, Luu \& Chen 1996; Jewitt Luu \& Trujillo 1998). The objects detected by ground-based searches have had magnitudes $20 \cdot 6<m_{R}<25 \cdot 0$ with the majority of objects having magnitudes $22<m_{R}<24$. Several surveys have covered larger areas of sky to brighter limiting magnitudes, but these surveys have not detected any Kuiper Belt objects. The size and magnitude distribution of Kuiper Belt objects brighter than $m_{R} \sim 21$ is therefore not well constrained.

Ideally one would like to continue previously successful surveys until larger objects are detected. However, all the successful surveys so far have used $1.5 \mathrm{~m}$ to $4 \mathrm{~m}$ telescopes at good sites that are heavily used by other astronomers. In this search, the compromise solution was to use a $1 \mathrm{~m}$ telescope with a large field of view. The disadvantage with using a smaller telescope is that the limiting magnitude is significantly brighter than that expected on a larger telescope.

While shallow surveys of large areas of sky have not detected Kuiper Belt objects, several have detected objects in orbits between Jupiter and Neptune (Centaurs). Only seven Centaurs have been detected which have well-determined orbits (Marsden 1997). Centaurs and Kuiper Belt objects have a slow apparent motion compared to main-belt asteroids and they are often described as slow moving objects (SMOs).

Table 1. Surveys for Kuiper Belt objects

The validity of the statistical detection of 29 objects by Cochran et al. (1995) is debatable (Jewitt, Luu \& Chen 1996; Brown, Kulkarni \& Liggett 1997) and it has not been included in the analysis of the sky surface density presented in this paper. The $m_{R}$ limit for Kowal (1989) is that given in Irwin, Tremaine \& Żytkow (1995), while the $m_{R}$ limit for Levison \& Duncan (1990) is that given by Levison (private communication). Only $50 \%$ of the Kuiper Belt objects at the magnitude limit of Jewitt, Luu \& Trujillo (1998) could be detected by that survey. A detailed discussion of previous surveys for Kuiper Belt objects can be found in Brown (1997). Another 22 Kuiper Belt objects have been detected (Marsden 1997) by surveys that have not been published in peer reviewed journals

\begin{tabular}{lccc}
\hline Reference & $\begin{array}{c}m_{R} \\
\text { limit }\end{array}$ & $\begin{array}{c}\text { Area } \\
\left(\square^{\circ}\right)\end{array}$ & Detections \\
\hline Tombaugh (1961) & $16 \cdot 8$ & 1530 & 0 \\
Kowal (1989) & $18 \cdot 5$ & 6400 & 0 \\
Luu \& Jewitt (1988) & $19 \cdot 5$ & 297 & 0 \\
Irwin et al. (1995) & $20 \cdot 0$ & 50 & 0 \\
Levison \& Duncan (1990) & $21 \cdot 0$ & $4 \cdot 9$ & 0 \\
Present work & $21 \cdot 0$ & $12 \cdot 0$ & 0 \\
Williams et al. (1995) & $22 \cdot 0$ & $0 \cdot 5$ & 1 \\
Jewitt et al. (1998) & $22 \cdot 5$ & $51 \cdot 5$ & 13 \\
Jewitt et al. (1996) & $23 \cdot 2$ & $4 \cdot 4$ & 3 \\
Irwin et al. (1995) & $23 \cdot 5$ & $0 \cdot 7$ & 2 \\
Luu \& Jewitt (1988) & $24 \cdot 0$ & $0 \cdot 34$ & 0 \\
Jewitt et al. (1996) & $24 \cdot 2$ & $3 \cdot 9$ & 12 \\
Jewitt \& Luu (1995) & $24 \cdot 8$ & $1 \cdot 2$ & 7 \\
Cochran et al. (1995) & $28 \cdot 6$ & $0 \cdot 0011$ & $29 ?$ \\
\hline
\end{tabular}




\section{Observations}

Four observing runs between April 1995 and April 1997 used the Mount Stromlo and Siding Spring Observatories 40-inch telescope to search for Kuiper Belt objects. The first three observing runs used a $2250 \times 1152$ CCD with a focal reducer, the Low Dispersion Survey Spectrograph (Colless et al. 1990), to increase the size of the field. The resulting pixel scale was $2 \cdot 1^{\prime \prime}$ per pixel, however the entire CCD was not illuminated; so the field was $0 \cdot 44 \square^{\circ}$. The $2 \cdot 1^{\prime \prime}$ pixel scale results in undersampling of stellar images so analysis techniques such as difference maps (Irwin, Tremaine \& Żytkow 1995) could not be used. The final observing run used a $2048 \times 2048$ thinned Tektronix CCD with a pixel scale of $0 \cdot 6^{\prime \prime}$ per pixel and a field of view of $0 \cdot 11 \square^{\circ}$. All observing runs used R-band filters as this is the most efficient band because of the solar to red object colours (Luu 1994; Luu \& Jewitt 1996) and the CCD wavelength response.

The apparent motion (in "/hr) near opposition of an object orbiting at distance $R$, where $R \gg 1 \mathrm{au}$, is given by

$$
\frac{d \theta}{d t} \simeq 148 \cdot 5 \mathrm{au} \times \frac{\cos \omega}{R}
$$

where $\omega$ is the angle from opposition. For an object just beyond Neptune, this results in an apparent motion of $\sim 3^{\prime \prime} /$ hr near opposition. This motion is detectable in a single night's observations so, where possible, all observations were done in a single night in dark time. This reduced problems caused by changing weather conditions and allowed for easier image analysis.

A minimum of three sets of $3 \times 400 \mathrm{~s}$ integrations of each target field were made at intervals of at least 2 hours. Integrations longer than half an hour were not possible due to trailing loss caused by the apparent motion of SMOs across the sky. Images were also taken of Landolt (1992) and Graham (1982) standards to provide photometric calibration.

All images were bias subtracted, flat fielded and blinked to search for candidates. Blinking was used along with less time consuming automated techniques as fainter candidates can be detected by eye (Jewitt and Luu 1995; Levison, private communication; Jewitt, private communication). Images (Irwin 1985), an image detection program, was used to detect objects and our own software was then used to select objects moving with a regular rate of motion. While not as efficient as blinking, this allowed candidates to be detected at the telescope, allowing rapid follow-up observations. Full details of the observation strategy and data reduction are presented in Brown (1997).

An $m_{R} \simeq 21$ SMO candidate was identified in three images taken on 25 April 1995. The $8 \cdot 3 \pm 0 \cdot 3^{\prime \prime} / \mathrm{hr}$ apparent motion of the candidate indicated that this object was not a member of the Kuiper Belt. It is possible that this object is a Centaur in an eccentric and inclined orbit but it is also possible that it is closer to Earth (Marsden, private communication). Astrometry for the candidate is given in Table 2 .

Table 2. Candidate astrometry

\begin{tabular}{lccc}
\hline UT date & Time & $\begin{array}{c}\text { RA }(\mathrm{J} 2000) \\
\pm 0 \cdot 7^{\prime \prime}\end{array}$ & $\begin{array}{c}\text { Dec }(\mathrm{J} 2000) \\
\pm 0 \cdot 7^{\prime \prime}\end{array}$ \\
\hline $25 / 4 / 95$ & 1108 & $140216 \cdot 99$ & $-124727 \cdot 4$ \\
$25 / 4 / 95$ & 1338 & $140215 \cdot 78$ & $-124714 \cdot 5$ \\
$25 / 4 / 95$ & 1636 & $140214 \cdot 45$ & $-124700 \cdot 7$ \\
\hline
\end{tabular}

A second $m_{R} \simeq 21 \mathrm{SMO}$ candidate was identified in three images taken on 24 April 1995. The candidate's apparent motion of $1 \cdot 8^{\prime \prime} / \mathrm{hr}$ corresponds to a distance of $\sim 55 \mathrm{au}$. Observations of the same field in April 1997 with the $2048 \times 2048$ CCD in good seeing detected a $m_{R} \sim 21$ star near the position where the candidate was detected and it is assumed that the candidate was produced by the star and noise.

Magnitude limits were determined by adding artificial SMOs to sets of images at random positions. The artificial SMOs were then detected by blinking and with automated techniques. The limiting magnitude of the $2250 \times 1152$ CCD images was $m_{R} \simeq 21$. The higher quantum efficiency and lower read-out noise of the $2048 \times 2048 \mathrm{CCD}$ resulted in a limiting magnitude of $m_{R} \simeq 21 \cdot 5$. Deeper limiting magnitudes were achieved for a small number of images where seeing was below $1 \cdot 5^{\prime \prime}$. The capability of the search to detect SMOs was also tested by recovering 2060 Chiron $\left(m_{R} \sim 16\right)$, $1995 \mathrm{GO}\left(m_{R} \sim 19\right)$ and $1995 \mathrm{DW}_{2}\left(m_{R} \simeq 21 \cdot 2\right)$.

Table 3. Sky surface density of Kuiper Belt objects

\begin{tabular}{lccl}
\hline$m_{R}$ & $\begin{array}{c}\text { Area } \\
\left(\square^{\circ}\right)\end{array}$ & Detections & \multicolumn{1}{c}{$\begin{array}{c}\Sigma \\
\mathrm{deg}^{-2}\end{array}$} \\
\hline $18 \cdot 5$ & 6800 & 0 & $<6 \cdot 8 \times 10^{-4}$ \\
$19 \cdot 5$ & 393 & 0 & $<1 \cdot 1 \times 10^{-2}$ \\
$21 \cdot 0$ & 69 & 2 & $3 \cdot 0 \pm_{2}^{3} \cdot 7^{\circ} \times 10^{-2}$ \\
$22 \cdot 0$ & $41 \cdot 6$ & 7 & $0 \cdot 19 \pm_{0}^{0} \cdot 11$ \\
$23 \cdot 2$ & $10 \cdot 2$ & 16 & $1 \cdot 6 \pm_{0}^{0} \cdot 4$ \\
$24 \cdot 2$ & $5 \cdot 1$ & 20 & $3 \cdot 9 \pm_{1}^{1} \cdot 1$ \\
$24 \cdot 8$ & $1 \cdot 2$ & 7 & $5 \cdot 8 \pm_{2}^{3} \cdot 3$ \\
\hline
\end{tabular}

\section{Luminosity Function}

The luminosity function of the Kuiper Belt has, until recently, not been well constrained near $m_{R} \sim 21$. This is due to the small areas covered by most surveys to limiting magnitudes $m_{R}>21$. Using the data from this survey and previous work, new constraints were determined on the luminosity function of the Kuiper Belt near $m_{R} \sim 21$. Table 3 shows the constraints on the sky surface density of Kuiper Belt objects using data from this work, Kowal (1989), Luu and Jewitt (1988), Levison and Duncan (1990), Jewitt and Luu (1995), Irwin, Tremaine \& Żytkow 
(1995), Jewitt, Luu \& Chen (1996) and Jewitt, Luu \& Trujillo (1998). Upper limits and error margins were calculated with Poisson statistics. Upper limits are $99 \%$ confidence limits and error margins have $68 \%$ confidence. It should be noted that the sky surface density does not always equal the number of detections divided by the survey area due to the decrease of detection efficiency with magnitude. In particular, Jewitt et al. (1998) has an $\sim 80 \%$ detection efficiency at $m_{R} \sim 21$, but this decreases to $\sim 60 \%$ by $m_{R} \sim 22$.

Pluto has been excluded from the analysis as it is 8 magnitudes brighter than any known Kuiper Belt object. As Table 3 includes data from surveys with different limiting magnitudes, the number of objects detected does not always increase with limiting magnitude.

Figure 1 is plot of the sky surface density of Kuiper Belt objects near the ecliptic. The detections of bright Kuiper Belt objects by Jewitt et al. (1998) indicate that a break in the luminosity of the Kuiper Belt is not required unless the photographic survey by Kowal (1989) had a high detection efficiency to the limiting magnitude stated by Kowal or Irwin et al. (1995). A CCD survey of $100 \square^{\circ}$ would provide a much improved estimate of the population of bright Kuiper Belt objects and possibly detect a break in the luminosity function.

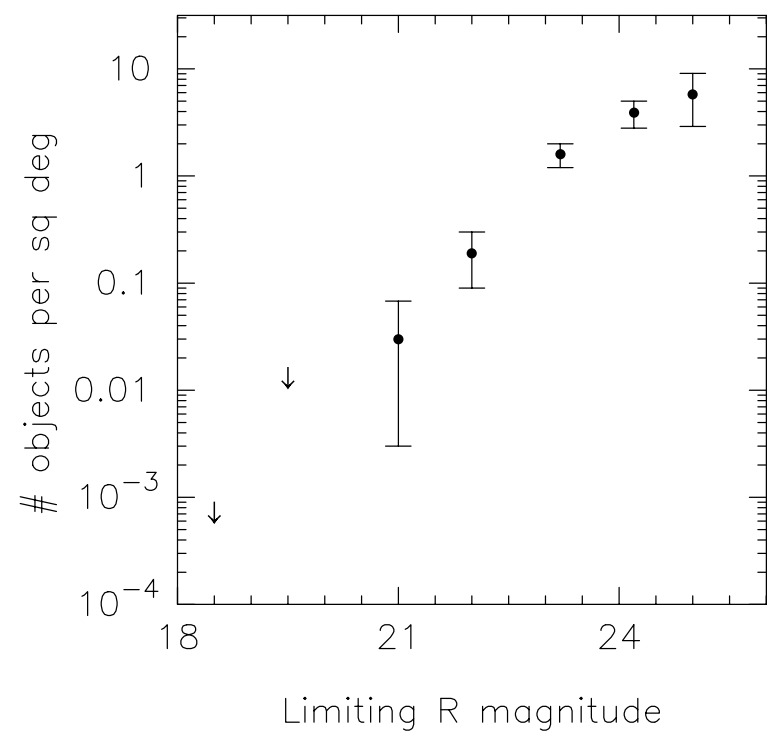

Figure 1-Sky surface density of Kuiper Belt objects.

\section{Conclusions}

A search of $12 \cdot 0 \square^{\circ}$ near the ecliptic to a limiting magnitude of $m_{R} \sim 21$ has been conducted for bright Kuiper Belt objects. This search and other surveys constrain the number of Kuiper Belt objects brighter than our limiting magnitude to $3 \cdot 0 \pm_{2}^{3} \cdot 8 \times 10^{-2}$ per $\square^{\circ}$. Surveys with the planned $8 \mathrm{k} \times 8 \mathrm{k}$ CCD on the Siding Spring 40-inch telescope should provide an improved estimate of the population of bright Kuiper Belt objects and further constrain the bright end of the luminosity function.

\section{Acknowledgments}

The authors would like to thank Chris Fluke, Michael Hicks and Thomas Irving for their assistance during observing with the Mount Stromlo and Siding Spring Observatories 40-inch telescope. We would also like to thank the Mount Stromlo and Siding Spring Observatories Time Allocation Committee for allocating several weeks of dark time on the 40-inch telescope for this survey.

\section{References}

Brown, M. E., Kulkarni, S. R., \& Liggett, T. J. 1997, ApJ, 490, L119

Brown, M. J. I. 1997, MSc thesis, University of Melbourne Cochran, A. L., Levison, H. F., Stern, S. A., \& Duncan, M. J. 1995, ApJ, 455, 342

Colless, M., Ellis, R. S., Taylor, K., \& Hook, R. N. 1990, MNRAS, 244, 408

Edgeworth, K. 1949, MNRAS, 109, 600

Graham, J. 1982, PASP, 94, 244

Irwin, M. 1985, MNRAS, 214, 575

Irwin, M., Tremaine, S., \& Żytkow, A. 1995, AJ, 110, 3082

Jewitt, D., \& Luu, J. 1993, Nature, 362, 730

Jewitt, D., \& Luu, J. 1995, AJ, 109, 1867

Jewitt, D., Luu, J., \& Chen, J. 1996, AJ, 112, 1225

Jewitt, D., Luu, J., \& Trujillo, C. 1998, AJ, 115, 2125

Kowal, C. 1989, Icarus, 77, 118

Kuiper, G. P. 1951, in Astrophysics, ed. J. A. Hynek (New York: McGraw-Hill), p. 357

Landolt, A. 1992, AJ, 104, 340

Levison, H. F., \& Duncan, M. J. 1990, AJ, 100, 1669

Luu, J. 1994, in Asteroids, Comets, Meteors 1993, ed. A. Milani et al. (Amsterdam: Kluwer), p. 31

Luu, J., \& Jewitt, D. 1988, AJ, 95, 1256

Luu, J., \& Jewitt, D. 1996, AJ, 111, 499

Marsden, B. G. 1997, Minor Planet Electronic Circular V25

Tombaugh, C. W. 1961, in Planets and Satellites, ed. G. P. Kuiper \& B. M. Middlehurst (University of Chicago Press), p. 12

Williams, I., O'Ceallaigh, D., Fitzsimmons, A., \& Marsden, B. G. 1995, Icarus, 116, 180 